\title{
Modelamiento matemático del proceso de deshidratado de china konoca (Xenophyllum poposum) por aire caliente
}

\author{
Mathematical modeling of the dehydrating process of china konoca (Xenophyllum \\ poposum) by hot air
}

Modelagem matemática do processo de desidratação da china konoca (Xenophyllum poposum) por ar quente

\author{
- Gustavo Adolfo Espinoza Calderón \\ gustavo.espinoza@unh.edu.pe \\ Código ORCID: 0000-0002-8299-9449
}

- Carmen Taipe Lucas

carmen.taipe@unh.edu.pe

Código ORCID: 0000-0003-1538-2753

\section{- Magdalena Taipe Yalli \\ magdalenataipeyalli@gmail.com \\ Código ORCID: 0000-0002-7243-3089}

Universidad Nacional de Huancavelica, Escuela Profesional de Ingeniería agroindustrial, Huancavelica-Perú

\section{RESUMEN}

Durante mucho tiempo las plantas medicinales fueron el principal recurso disponible para tratar enfermedades. Por esta razón fueron desarrollándose numerosos estudios sobre esas plantas e investigaciones sobre el uso de sus productos y derivados. La china konoca (Xenophyllum poposum) es una planta nativa que crece de manera silvestre en los Andes peruanos a más de 4400 metros sobre el nivel del mar, es muy utilizada como una infusión natural para la digestión. El propósito de este trabajo fue realizar un modelado matemático durante el proceso de deshidratado de la china konoca (Xenophyllum poposum). Para ello, se efectuó la evaluación de cinética de deshidratado de esta planta a $60^{\circ} \mathrm{C}$ y $80^{\circ} \mathrm{C}$, durante 480 y 600 minutos, en donde se hizo las mediciones de pérdida de humedad. Se obtuvieron gráficas de velocidad de deshidratado en función del tiempo, temperatura y la humedad, con estos datos se realizó un modelado matemático de cada tratamiento para describir la tendencia en las curvas de deshidratado. Resultando un modelo polinómico de segundo grado el que mostró mejor bondad de ajuste en la cinética de secado por aire caliente, $\% \mathrm{H}=\mathrm{a}-\mathrm{b} \theta+\mathrm{c} \theta$ 2. Además, se hizo el modelamiento para predecir el tiempo de equilibrio en el decrecimiento de humedad, derivando la ecuación anterior, $\theta=\mathrm{b} / 2 \mathrm{c}$.

Palabras clave: Xenophyllum poposum; china konoca; deshidratación; modelo matemático

\section{ABSTRACT}

For a long time medicinal plants were the main resource available to treat diseases. For this reason, numerous studies were carried out on these plants and investigations on the use of their products and derivatives. The Chinese konoca (Xenophyllum poposum) is a native plant that grows wild in the Peruvian Andes at more than 4400 meters above sea level, it is widely used as a natural infusion for digestion. The purpose of this work was to perform a mathematical modeling during the dehydration process of the Chinese konoca (Xenophyllum poposum). For this, the dehydration kinetics evaluation of this plant was carried out at $60^{\circ} \mathrm{C}$ and $80^{\circ} \mathrm{C}$, during 480 and 600 minutes, where the moisture loss measurements were made. Dehydration speed graphs were obtained as a function of time, temperature and humidity, with these data a mathematical modeling of each treatment was performed to describe the trend in the dehydration curves. Resulting in a second degree polynomial model which showed the best goodness of fit in the kinetics of hot air drying, $\% \mathrm{H}=\mathrm{a}-\mathrm{b} \theta+\mathrm{c} \theta 2$. In addition, the modeling was done to predict the equilibrium time in the decrease humidity, deriving the previous equation, $\theta=\mathrm{b} / 2 \mathrm{c}$.

Key words: Xenophyllum poposum; china konoca; dehydration; mathematical model

\section{RESUMO}

Por muito tempo, as plantas medicinais foram o principal recurso disponível para o tratamento de doenças. Por esse motivo, inúmeros estudos foram realizados com essas plantas e pesquisas sobre o uso de seus produtos e derivados. A convoca chinesa (Xenophyllum poposum) é uma planta nativa que cresce de forma selvagem nos Andes peruanos a mais de 4400 metros acima do nível do mar, é amplamente utilizada como uma infusão natural para a digestão. 0 objetivo deste trabalho foi realizar uma modelagem matemática durante o processo de desidratação da konoca chinesa (Xenophyllum poposum). Para isso, a avaliação cinética de desidratação desta planta foi realizada a $60^{\circ} \mathrm{C}$ e $80^{\circ} \mathrm{C}$, durante 480 e 600 minutos, onde foram feitas as medições de perda de umidade. Gráficos de velocidade de desidratação foram obtidos em função do tempo, temperatura e umidade, com esses dados foi realizada uma modelagem matemática de cada tratamento para descrever a tendência das curvas de desidratação. Resultando em um modelo polinomial de segundo grau que apresentou o melhor ajuste na cinética de secagem a ar quente, $\% \mathrm{H}=\mathrm{a}-\mathrm{b} \theta$ + c $\theta$ 2. Além disso, a modelagem foi feita para prever o tempo de equilíbrio na diminuição da umidade, derivando a equação anterior, $\theta=b$ / $2 c$.

Palavras-chave: Xenophyllum poposum; china konoca; desidratação; modelo matemático 


\section{INTRODUCCIÓN}

Huancavelica en Perú, cuenta con una diversidad de plantas medicinales entre ellas está la china konoca muy utilizada por sus propiedades curativas. La falta de conocimiento de las propiedades curativas de esta planta hace que no se valore la diversidad que es existente en la región sabiendo sus propiedades, se podría contribuir en el alivio de malestares como el dolor estomacal, soroche o "mal viento". Gracias a sus efectos curativos, está siendo usada en la población de manera artesanal sin ser investigada. En el centro poblado del Barrio San Felipe, distrito de Pilpichaca, región Huancavelica, esta planta habita todo el año, con una mayor producción en los meses de enero hasta abril, es utilizada por los pobladores en infusiones, como medicina alternativa, principalmente para controlar los dolores estomacales (1).

La china konoca (Xenophyllum poposum) es una planta andina que crece en Perú, en zonas de climas fríos, como en el barrio San Felipe, distrito de Pilpichaca, departamento de Huancavelica. Sin embargo, esta planta que crece de manera silvestre, todavía no ha sido utilizada en la agroindustria. Es muy apreciada por aquellas personas que conocen esta planta y la usan, debido a sus cualidades curativas, por su sabor y aroma intenso (2).

El propósito de esta investigación es obtener un producto deshidratado, para preservar los compuestos bioactivos, al terminar las etapas de procesamiento en la china konoca, ya que es beneficioso para el consumo humano (3).

En la actualidad existen escasos conocimientos sobre esta planta andina, la cual es consumida por los pobladores de la zona sin conocimiento exacto de sus compuestos, únicamente por sus propiedades curativas, para el dolor estomacal, "mal de viento" y soroche, lo cual conlleva a realizar este trabajo de investigación (4). Para ello se realizó la deshidratación de la china Konoca por aire caliente mediante estufa, evaluando el efecto del tiempo y temperatura de deshidratado que permitan conservar en los posible las características fisicoquímicas y valor bioactivo. Se espera, mediante este trabajo, lograr un producto con mayor tiempo de vida útil, para aplicarlo a nivel comercial en el mercado regional, nacional e internacional (5).

El presente artículo tiene como objetivo principal evaluar el efecto del tiempo y temperatura del deshidratado de la china konoca (Xenophyllum poposum) en las características fisicoquímicas (6) y realizar un modelamiento matemático con las curvas de perdida de humedad durante el secado por aire caliente (7).

El presente trabajo de investigación, tiene mucha importancia para facilitar o dar a conocer sobre el efecto del tiempo y temperatura en el deshidratado en bandejas sobre las características fisicoquímicas de la china konoca (Xenophyllum poposum). Que a su vez esta planta es muy utilizada en las zonas donde crece, debido a que tiene efectos beneficiosos en aliviar el dolor estomacal, soroche y el mal viento que habita en el departamento de Huancavelica, esta planta posee una reconocida utilización en problemas cardiovasculares, así como también, para atenuar dolores estomacales. Por lo tanto, tiene como fin dar una alternativa, a las necesidades de transformación de plantas nativas (8). Por lo que es necesario promover y desarrollar la potencialidad de este producto, para lo cual se realizará el método de deshidratado para su conservación, como una alternativa tecnología, conociendo el estudio 
básico de la variación de efecto del tiempo y temperatura en el deshidratado en bandejas sobre las características fisicoquímicas $\mathrm{y}$ modelo matemático de deshidratado a la china konoca (Xenophyllum poposum), en relación a los niveles altitudinales donde se desarrolla (9).

Se considera necesario aprovechar las bondades de esta planta que hasta la fecha no ha tenido aplicaciones en el campo agroindustrial y farmacéutico. Además, con los resultados finales de este proyecto de investigación, se brindará información básica para realizar diferentes procesos agroindustriales de la especie de (Xenophyllum poposum) (10).

\section{MATERIALES Y MÉTODOS}

Se realizó una descripción del deshidratado de china konoca en donde llevó a cabo el proceso de recepción y pesado, también se recogió las hojas de china konoca fresca luego se hizo un control visual de la materia prima; se pesó, registró y verificó el total. Seguidamente se seleccionó separando las muestras que presentaron deterioro $\mathrm{y}$ daño físico; se llevó a cabo el lavado de las hojas el cual fue manual para quitar la tierra y otras impurezas, seguidamente, las hojas fueron oreadas para eliminar el agua que quedo durante el lavado, se continuó con la deshidratación colocando las muestras oreadas en bandejas dentro de un deshidratador a una temperatura de $60^{\circ} \mathrm{C}$ y $80^{\circ} \mathrm{C}$ entre 480 y 600 minutos, para luego conseguir el envasado y pesado de las muestras deshidratadas en bolsas.

\section{Cinética de deshidratado de la china konoca}

La caracterización de cinéticas de deshidratado se desarrolló graficando la evolución de la pérdida de humedad de la china konoca en función del tiempo hasta que llega al equilibrio de deshidratado, para las diferentes temperaturas de estudio y las velocidades de deshidratado, donde se explican el suceso de los periodos correspondientes. $(11,12)$.

\section{Modelado matemático de la cinética de deshidratado}

El modelado de cinética de deshidratado se realizó mediante un ajuste matemático, el cual puede ser polinómico, exponencial, logarítmico, etc. Para esto se elige el modelo de mayor arreglo tomando en cuenta el coeficiente de determinación (R2), se correlaciona los valores experimentales, de ganancia de perdida de humedad versus tiempo, para obtener expresiones empíricas a partir de análisis de regresión (13).

Dónde:

$$
\% H=f(\theta)
$$

- $\% \mathrm{H}=$ pérdida de humedad

- (f) $\theta=$ modelo matemático en función al tiempo

\section{Procedimiento de deshidratado}

Para el proceso de deshidratado de la china konoca se trabajó en base a lo reportado por (14), con las siguientes operaciones unitarias: 


\section{CHINA KONOCA}

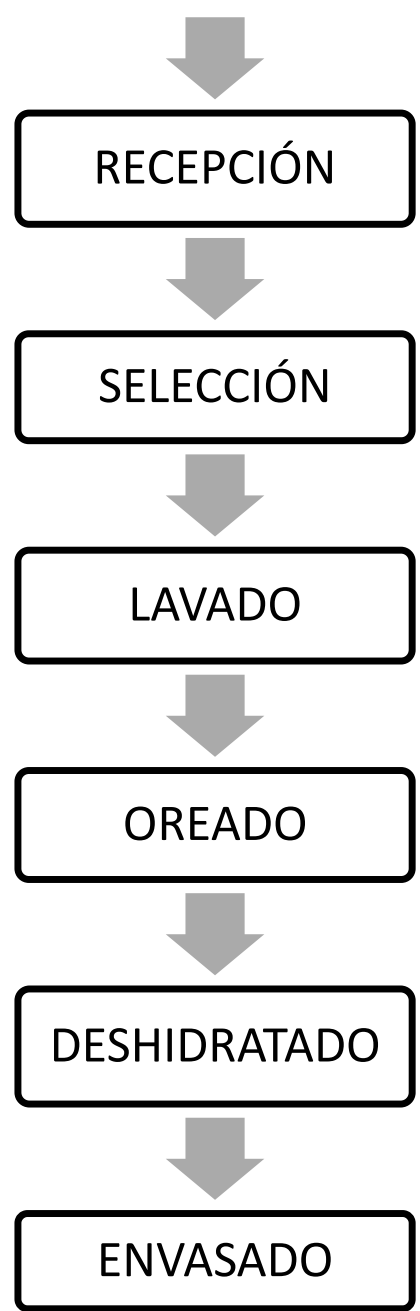

Determinación de humedad de la china konoca

RESULTADOS

Tabla 3 Contenido de humedad y solidos totales de la china konoca

\begin{tabular}{cc}
\hline Producto & \% Humedad \\
\hline china konoka & $55.0 \% \pm 0.13$ \\
\hline
\end{tabular}

\section{Caracterización de las curvas de deshidratado}

El desarrollo del deshidratado de la muestra de china konoca se muestran en la Figuras 2 y 3 a través de las curvas de deshidratado a las temperaturas de $60^{\circ} \mathrm{C}$ y $80^{\circ} \mathrm{C}$, por un tiempo de 480 y 600 minutos. 


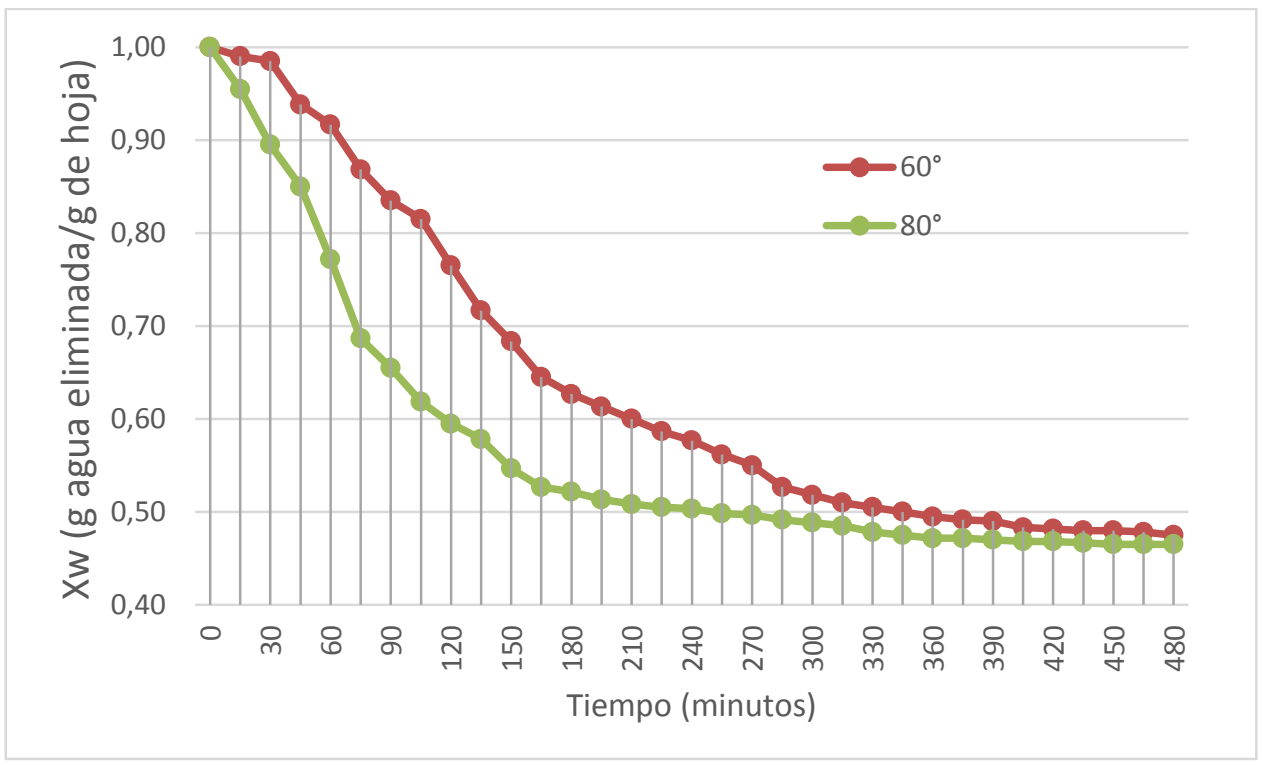

Figura 2. Curva de deshidratado a 60 y 80 durante 480 minutos.

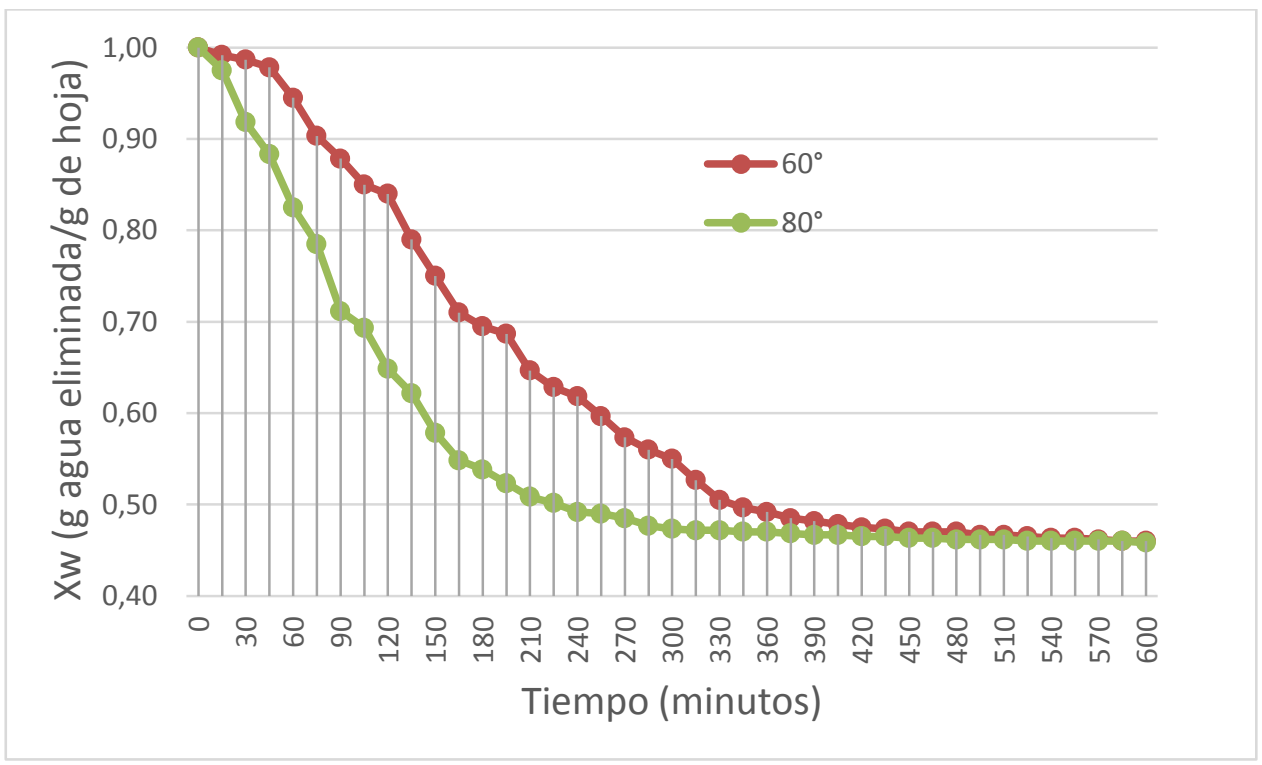

Figura 3. Curva de deshidratado a $60^{\circ}$ y $80^{\circ}$ durante 600 minutos.

\section{Modelamiento matemático para la pérdida de humedad de la china konoca}

Para realizar el modelo matemático de pérdida de humedad en la china konoca, se elaboró la curva de humedad versus tiempo de deshidratado para los 4 tratamientos, como se muestra en las Figura 4 y 5. 


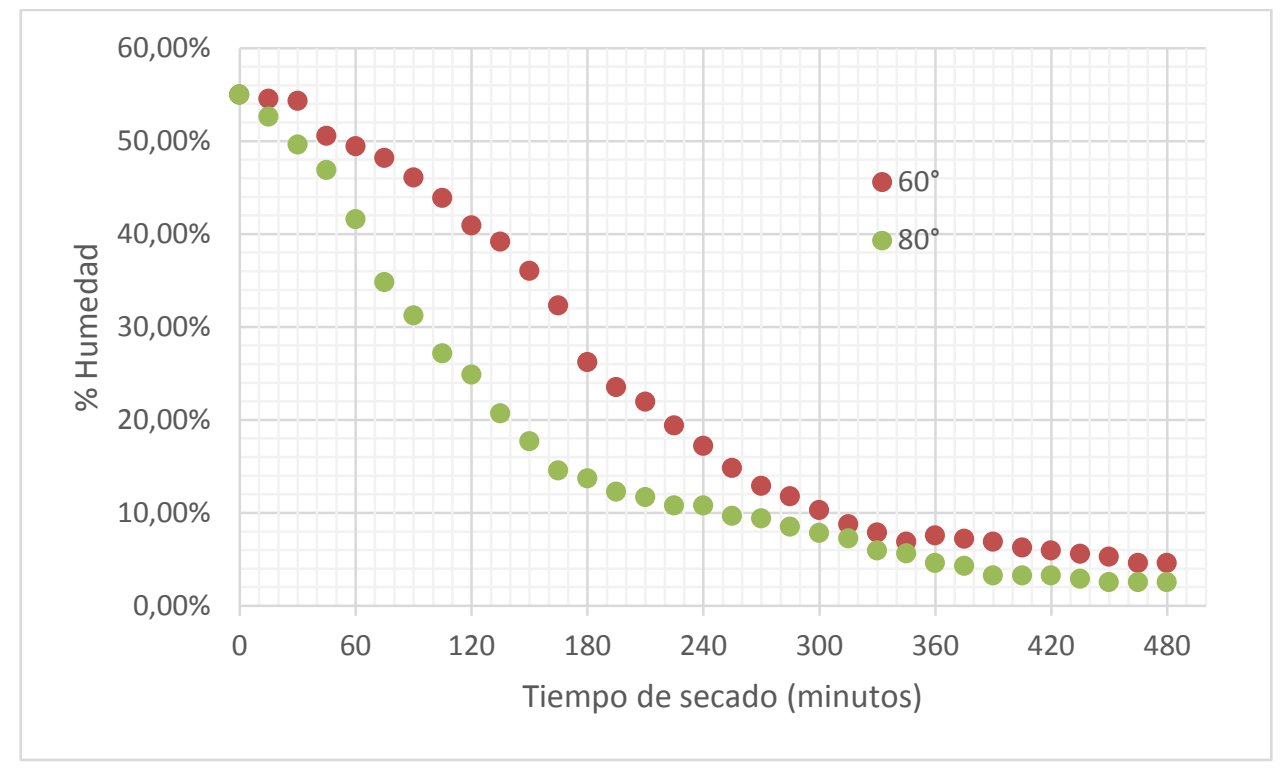

Figura 4. Datos experimentales para la pérdida de humedad en hojas de china konoca por 480 minutos a las temperaturas de 60 y $80^{\circ} \mathrm{C}$.

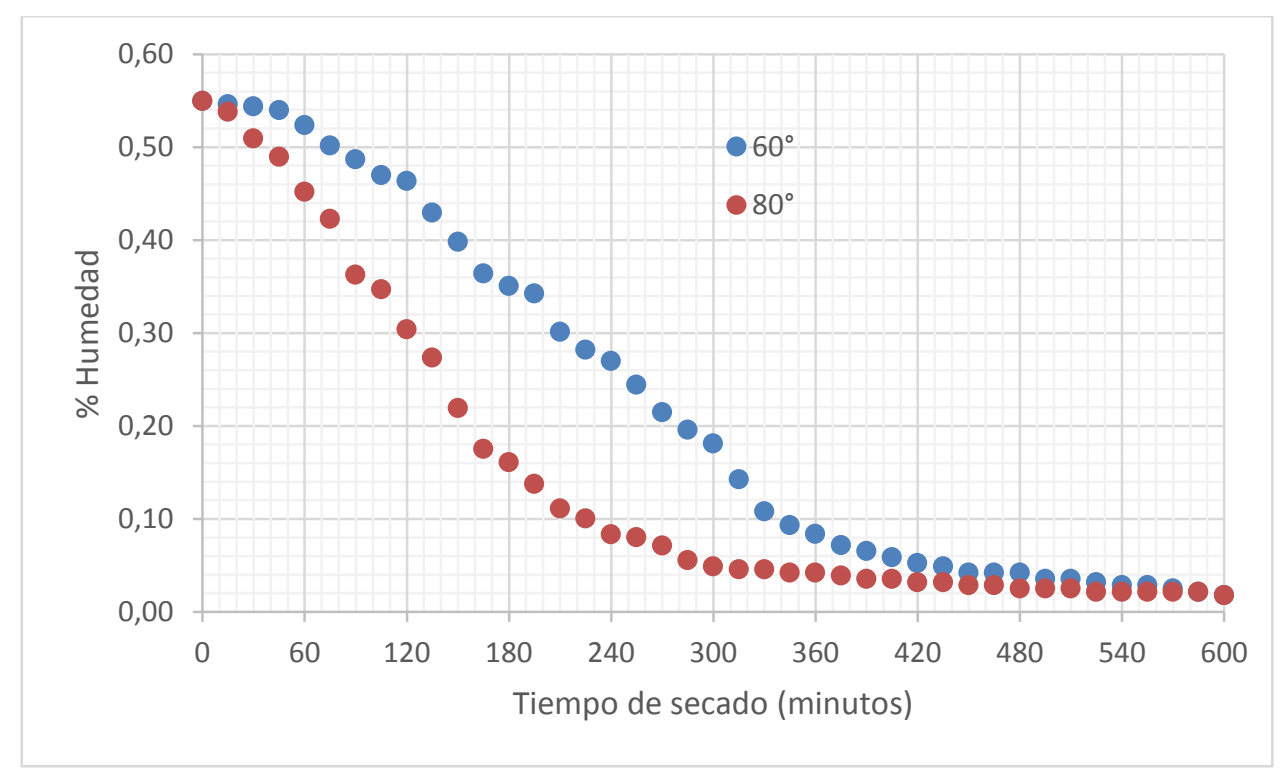

Figura 5. Datos experimentales para la pérdida de humedad en hojas de china konoca por 600 minutos a las temperaturas de 60 y $80^{\circ} \mathrm{C}$.

Se tomaron los datos obtenidos para el modelo matemático de pérdida de humedad en la china konoca, se eligió el polinomio de grado 2, por tener un mayor ajuste (R2> 0.97), comparado con el modelo logarítmico, potencial, exponencial y lineal. Para un tiempo $\theta$ la formula general es:

$$
\% \mathrm{H}=\mathrm{a}-\mathrm{b} \theta+\mathrm{c} \theta^{2}
$$




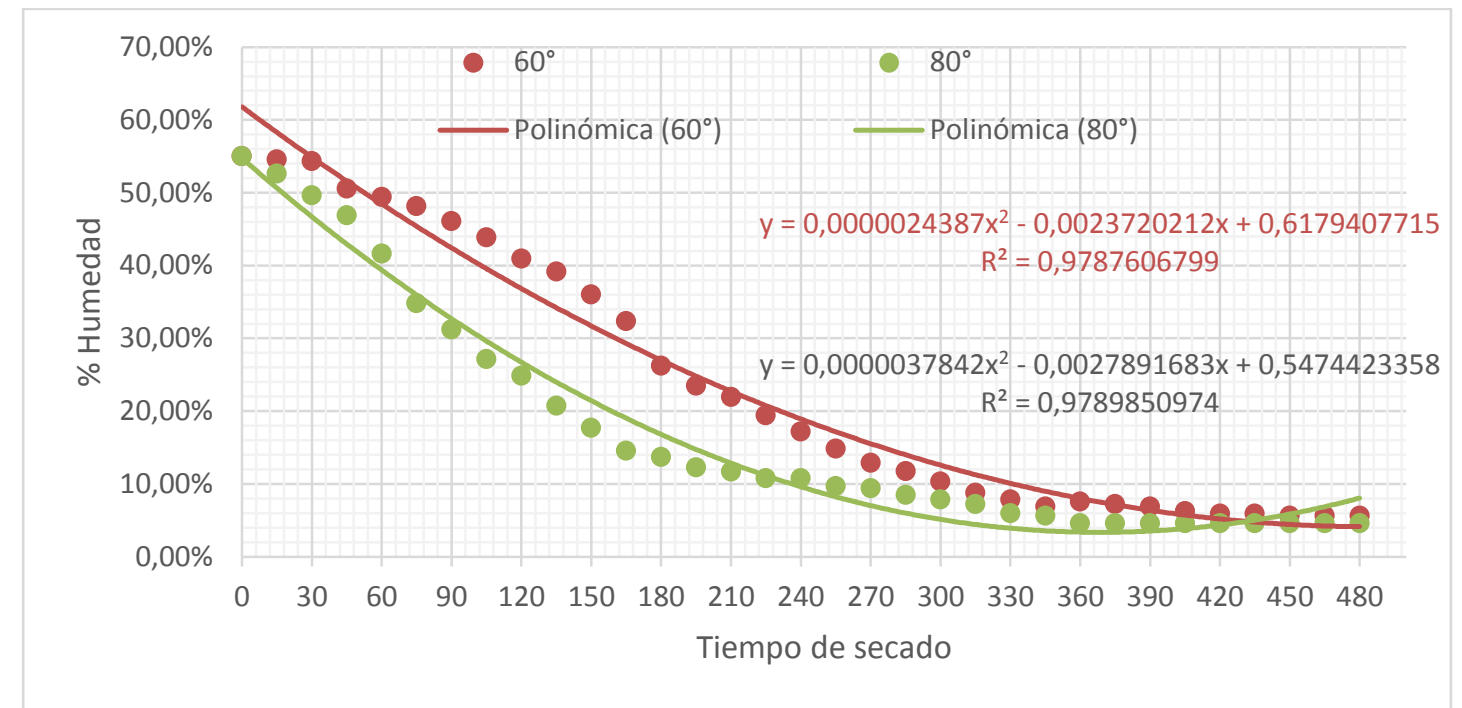

Figura 6. Ajuste polinómico de segundo grado a los datos experimentales de pérdida de humedad en china konoca a 60 y $80^{\circ} \mathrm{C}$ durante 480 minutos.

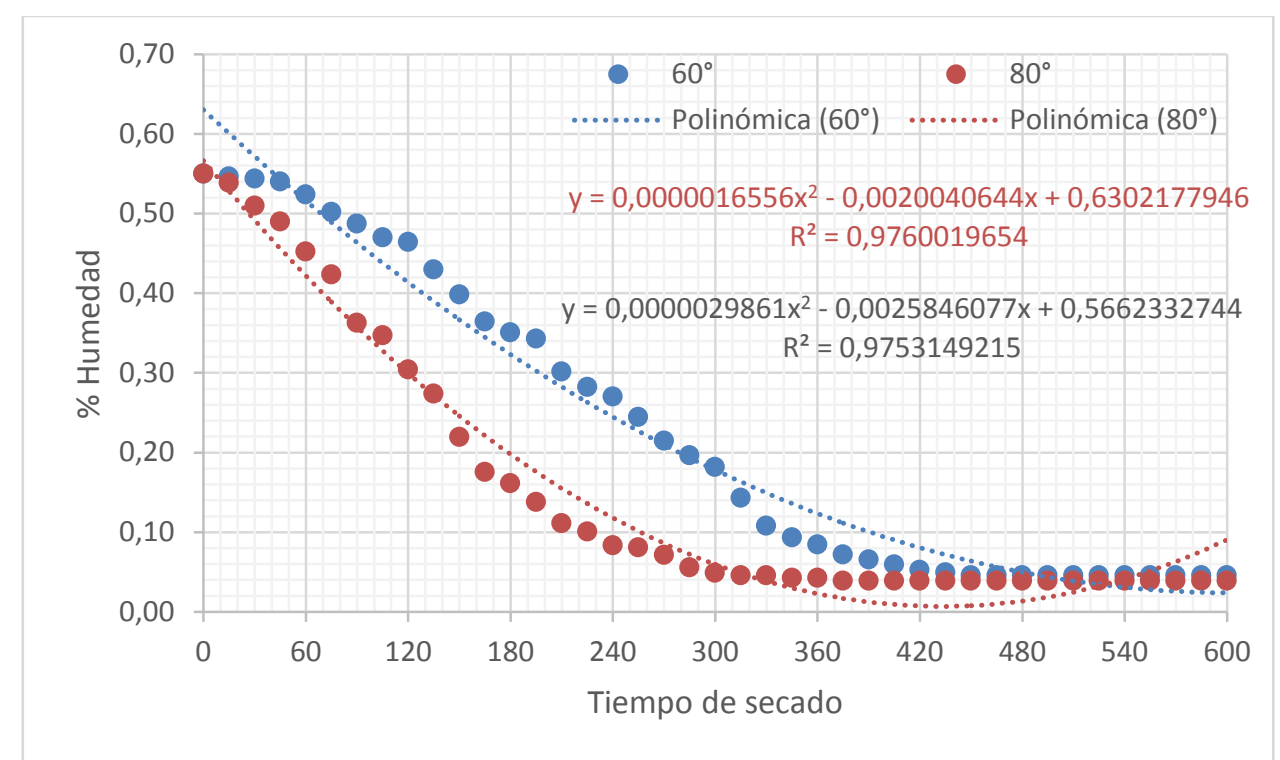

Figura 7. Ajuste polinómico de segundo grado a los datos experimentales de pérdida de humedad en china konoca a 60 y $80^{\circ} \mathrm{C}$ durante 600 minutos.

\section{Modelos matemáticos}

Para un tiempo de deshidratado $\theta$ y una pérdida de humedad $(\% \mathrm{H})$ en hojas de china konoca según la formula general:

$$
\% \mathrm{H}=\mathrm{a}-\mathrm{b} \theta+\mathrm{c} \theta^{2}
$$

Alfa, Revista de Investigación en Ciencias Agronómicas y Veterinarias Vol. 5, JNro. 13, Enero - abril 2021 


\section{Para $60^{\circ} \mathrm{C}$ por 480 minutos:}

$\% \mathrm{H}=0.6179-0.0023 \theta+2.4 \times 10^{-6} \theta^{2}$

$\mathrm{R}^{2}=0,9788$

\section{Para $80{ }^{\circ} \mathrm{C}$ por 480 minutos:}

$\% \mathrm{H}=0.5474-0.0028 \theta+3.8 \times 10^{-6} \theta^{2}$

$\mathrm{R}^{2}=0,9789$

\section{Para $60{ }^{\circ} \mathrm{C}$ por 600 minutos:}

$\% \mathrm{H}=0.6302-0.0020 \theta+1.6 \times 10^{-6} \theta^{2}$

$\mathrm{R}^{2}=0,9760$

\section{Para $80{ }^{\circ} \mathrm{C}$ por 600 minutos:}

$\% \mathrm{H}=0.5662-0.0026 \theta+2.9 \times 10^{-6} \theta^{2}$

$\mathrm{R}^{2}=0,9753$

Tabla 2. Valores $\mathrm{R}^{2}$ para el modelado polinómico de grado 2 .

\begin{tabular}{ccc}
\hline Tiempo & Temperatura & $\mathbf{R}^{\mathbf{2}}$ \\
\hline \multirow{2}{*}{480 minutos } & $60^{\circ} \mathrm{C}$ & 0,9788 \\
& $80{ }^{\circ} \mathrm{C}$ & 0,9789 \\
600 minutos & $60^{\circ} \mathrm{C}$ & 0,9760 \\
& $80{ }^{\circ} \mathrm{C}$ & 0,9753 \\
\hline
\end{tabular}

Determinación del tiempo de equilibrio teórico de la pérdida de humedad de la china konoca

Se derivó la pérdida de humedad simbolizada por $\% \mathrm{H}$ respecto a un tiempo $\theta$ para encontrar el tiempo de equilibrio teórico de pérdida de agua:

$$
\begin{gathered}
d(\% H) / d(\theta)=d\left(a-b \theta+c \theta^{2}\right) / d(\theta)=0 \\
-b+2 c \theta=0 \\
\theta=b / 2 c
\end{gathered}
$$

Tabla 5. Valores de humedad de equilibrio.

\begin{tabular}{cccc}
\hline Tratamiento & b & c & b/2c \\
\hline $60^{\circ}-480 \mathrm{~min}$ & 0.0024 & 0.0000024 & 486 \\
$80^{\circ}-480 \mathrm{~min}$ & 0.0028 & 0.0000038 & 368 \\
$60^{\circ}-600 \mathrm{~min}$ & 0.0020 & 0.0000016 & 605 \\
$80^{\circ}-600 \mathrm{~min}$ & 0.0026 & 0.0000029 & 432 \\
\hline
\end{tabular}




\section{Discusión}

El resultado obtenido para el análisis de humedad en la china konoca (Xenophyllum poposum), con un contenido de 55\%, nos indica que es un producto susceptible a deterioro microbiano, razón por la cual es necesario realizar el proceso de deshidratado y disminuirá la actividad de agua para prolongar su tiempo de vida útil como infusión u otro producto derivado. Debido a que es una planta, de la cual no se encontraron estudios de investigación, los datos fueron comparados con la especie Xenophyllum dactylophyllum, planta del mismo género de la china konoca, estudiada por (5), quien reportó un porcentaje de humedad de $48.6 \%$ para este vegetal, hallando similitudes de humedad entre estas dos especies. Por otra parte (6) realizó un estudio con la punamuña, una hierba de $60 \%$ de humedad, en la que también hizo un deshidratado como método de conservación y para uso como infusión

La velocidad de deshidratado, de la china konoca, disminuye a medida que la humedad de la hoja decrece, y para describir este fenómeno se desarrolló el modelado matemático, considerando la relación, perdida de humedad versus tiempo de deshidratado. Se ajustó los datos experimentales de perdida de humedad a un modelo polinómico de segundo grado, este modelo se adecuó satisfactoriamente a los datos obtenidos durante el deshidratado, lo que revela que sirve para pronosticar la pérdida de humedad durante el deshidratado de hojas de china konoca a las condiciones trabajadas de temperatura y tiempo de deshidratado. Otros autores también realizaron modelados a la cinética de deshidratado como (6), en este caso se encontró que el modelo de deshidratado de la punamuña, que mejor se ajustó para las temperaturas de $40^{\circ} \mathrm{C}, 50^{\circ} \mathrm{C}$ y $60^{\circ} \mathrm{C}$ fue el de Thompson presentando valores $\mathrm{R}^{2}$ mayores a 0.94 y la modelo de Page con $\mathrm{R}^{2}$ mayores a 0.985. Estos valores de $\mathrm{R}^{2}$, que muestran el nivel de ajuste del modelado matemático, están acorde a los resultados obtenidos con la china konoca, con valores de $\mathrm{R}^{2}$ mayores a 0.97 .

Asimismo, mediante el modelado matemático con polinomios de segundo grado se pudo hallar el tiempo teórico de equilibrio durante el deshidratado de la china konoca. Obteniendo valores muy similares al tiempo de equilibrio real, los datos hallados arrojan que el menor tiempo de equilibrio fue de 370 minutos para el deshidratado a $80^{\circ} \mathrm{C}$ durante 480 minutos, y el mayor tiempo fue de 605 minutos para el deshidratado a $60^{\circ}$ durante 600 minutos.

Con estos datos se verifica, que la velocidad de deshidratado es apreciablemente menor, lo que aumenta su capacidad para absorber agua, favoreciendo la remoción de humedad, donde la humedad inicial es $55 \%$ en la muestra de la china konoca, hacemos que esto representa al $100 \%$ para las gráficas correspondientes. Así se puede corroborar que la merma de humedad en las hojas de china konoca depende tanto del tiempo como de la temperatura del aire de deshidratado.

\section{CONCLUSIONES}

Se evaluó el efecto del tiempo y temperatura de deshidratado en las características fisicoquímicas, de la china konoca. (Xenophyllum poposum) encontrando que la mayor perdida y ganancia de solidos totales fue para el tratamiento a $80^{\circ} \mathrm{C}$ durante 480 minutos, tiempo en el cual se llegó a la humedad de equilibrio. 
Se realizó un modelado matemático de la cinética de deshidratado de la china konoca para evaluar el efecto del tiempo $y$ temperatura encontrando que el modelo polinómico de segundo grado fue el que mejor se ajustó a la perdida de humedad de esta planta.

\section{REFERENCIAS BIBLIOGRÁFICAS}

1. Organización Mundial de la Salud (OMS). Estrategia de la OMS sobre Medicina Tradicional 2014-2023. (2013). Disponible en www.who.int.

2. Femenia J. Flora del Famatina. Revista Chilecito - La Rioja - Argentina (2007).

3. Apumayta $\mathrm{P}$, y Jorge 0 . Caracterización de los componentes bioactivos y la aceptabilidad organoléptica del filtrante a base de chachacoma (Senecio graveolens). Tesis de la Universidad Nacional de Huancavelica. Huancavelica (Perú). (2015).

4. Yarupaitán Genaro AJ. Flora silvestre de los Andes centrales del Perú: un estudio en la zona de Quilcas, Junín. Rev. Perú biol. 2003. Disponible

en: http://www.scielo.org.pe/scielo.php?script=s ci_arttext\&pid=S172799332003000200006\&l ng=es.

5. Quispe R. Efecto cicatrizante del extracto hidroalcohólico de las hojas de Xenophyllum dactylophyllum "conoca". Tesis de la Facultad de Ciencias de la Salud. Universidad Nacional de San Cristóbal de Huamanga (2017).

6. Monares H. Deshidratación de la punamuña (satureja boliviana) en secador de bandejas. Tesis de la Facultad de Ingeniería de la Universidad Nacional José María Arguedas. Andahuaylas - Perú (2015).

7. Arends, K. y Gutiérrez, V. Características de secado y modelado matemático para el bagazo de caña de azúcar tratado y no tratado en una planta piloto PDA. Tesis de grado. Facultad de Ing. Química. Universidad Rafael Urdaneta, Maracaibo Edo. Zulia. Venezuela (2008).
8. Moreno S. Evaluación del efecto de la temperatura de secado en las hojas del pachataya - pampataya. Tesis de la Facultad de Ingeniería de la Universidad Nacional José María Arguedas. Andahuaylas - Perú (2017).

9. Jinde A. Efecto de la temperatura y tiempo de secado en las propiedades físicas, químicas y microbiológicas de cuatro hortalizas: col de repollo (Brassica oleracea var. capitata cv. bronco), col morada (Brassica oleracea var. capitata f. rubra), lechuga iceberg tipo salinas (Lactuca sativa var. capitata) y espinaca (Spinacia oleracea L.), troceadas con previa aplicación de aceite esencial de canela (Cinnamomum zeylanicum). Tesis de la carrera de Ingeniería en Alimentos. Universidad Técnica de Ambato. Ecuador (2014).

10. Tonguino Borja MI. Determinación de las Condiciones Óptimas para la deshidratación de dos plantas aromáticas; Menta (mentha piperita l) y Orégano (origanum vulgare (), 2011 Disponible en: http://repositorio.utn.edu.ec/handle/123456 789/385

11. Pineda M, Chancon A, y Cordero G. Efecto sobre las condiciones de secado sobre la cinética de deshidratación de las hojas de morera (Muros alba). Agronomía mesoamericana, (2009).

12. Erazo D. Estudio del efecto de la deshidratación por aire sobre la capacidad antioxidante de la flor de jamaica (hibiscus sabdariffa l.). Universidad Tecnológica Equinoccial facultad de Ciencias de la Ingeniería e Industrias carrera de Ingeniería de Alimentos. Quito (Ecuador) (2016).

13. Castillo B, Mejía G, Castillo M, Gutiérrez C. y Quiroz R. Modelado matemático del secado de hojas de orégano (Plectranthus amboinicus) utilizando tecnologías directas e indirectas. Revista Académica de la Facultad de Ingeniería, Universidad Autónoma de Yucatán (2020). 
14. Aliaga E, \& Acevedo J. Factores para el procesamiento de la manzanilla común en la industria peruana de infusiones. Ingeniería
Industrial (2018); (036),

213-239. https://doi.org/10.26439/ing.ind2018.n036. 2455 